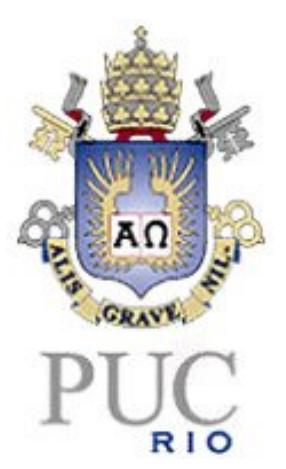

Antonio Fernando de Castro Vieira

\author{
Análise da Média e Dispersão em \\ Experimentos Fatoriais não Replicados para \\ Otimização de Processos Industriais
}

Tese de Doutorado

Tese apresentada como requisito parcial para obtenção do grau de Doutor pelo Programa de Pós-Graduação em Engenharia de Produção do Departamento de Engenharia Industrial da PUCRio.

Orientador: Prof. Eugênio Kahn Epprecht

Rio de Janeiro

Setembro de 2004 


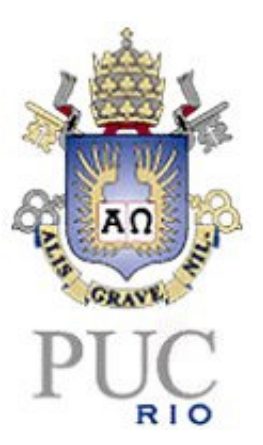

Antonio Fernando de Castro Vieira

\title{
Análise da Média e Dispersão em Experimentos Fatoriais não Replicados para Otimização de Processos Industriais
}

\begin{abstract}
Tese apresentada como requisito parcial para obtenção do grau de Doutor pelo Programa de Pós-Graduação em Engenharia de Produção do Departamento de Engenharia Industrial do Centro Técnico Cientifico da PUC-Rio. Aprovada pela Comissão Examinadora abaixo assinada.
\end{abstract}

Prof. Eugênio Kahn Epprecht Orientador Departamento de Engenharia Industrial - PUC-Rio

Prof. Sueli Aparecida Mingoti Departamento de Estatística - UFMG

Prof. Flávio Sanson Fogliatto

Departamento de Engenharia Produção e Transportes - UFRGS

Prof. Antônio Carlos Monteiro Ponce de Leon Instituto de Medicina Social - UERJ

Prof. Alvaro de Lima Veiga Filho Departamento de Engenharia Elétrica - PUC-Rio

Prof. José Eugenio Leal Coordenador(a) Setorial do Centro Técnico Científico - PUC-Rio 
Todos os direitos reservados. É proibida a reprodução total ou parcial do trabalho sem autorização da universidade, do autor e do orientador.

\section{Antonio Fernando de Castro Vieira}

Graduou-se em Engenharia Mecânica em 1968, pela Universidade Federal de Pernambuco, e concluiu o Mestrado em Engenharia Industrial na PUC-RIO em 1970. É professor do Departamento de Engenharia Industrial na PUC-RIO desde 1970. Exerceu atividades de análise de pesquisa operacional e operações de produção em diversas empresas.

Ficha catalográfica

Vieira, Antonio Fernando de Castro
Análise da média e dispersão em experimentos
fatoriais não replicados para otimização de processos
industriais / Antonio Fernando de Castro Vieira ; orientador:
Eugênio Kahn Epprecht. - Rio de Janeiro : PUC-Rio,
Departamento de Engenharia Industrial, 2004.
204 f. : il. ; 30 cm
Tese (doutorado) - Pontifícia Universidade
Católica do Rio de Janeiro, Departamento de Engenharia
Industrial.
Inclui referências bibliográficas
1. Engenharia industrial - Teses. 2. Qualidade.
generalizados. I. Epprecht, Eugênio Kahn. II. Pontifícia
Universidade Católica do Rio de Janeiro. Departamento de
Engenharia Industrial. III. Título.

CDD: 658.5 


\section{Resumo}

Vieira, Antonio Fernando de Castro Análise da Média e Dispersão em Experimentos Fatoriais não Replicados para Otimização de Processos Industriais. Rio de Janeiro, 2004. 204 p. Tese de Doutorado - Departamento de Engenharia Industrial, Pontifícia Universidade Católica do Rio de Janeiro.

Esta tese reúne as técnicas estatísticas indicadas para a modelagem da média e da dispersão das características de qualidade de processos e produtos, em experimentos fatoriais não replicados, resultando na definição de um roteiro integrado e detalhado de análise. A motivação vem de que, apesar de haver várias publicações sobre regressão linear clássica, modelos lineares generalizados (MLG), transformação da resposta e planejamento de experimentos, não existe um texto que reúna e descreva em detalhe todos os aspectos da modelagem da média e da dispersão em experimentos fatoriais. Os poucos textos sobre esse assunto não descrevem vários aspectos importantes em estudos dessa natureza, por exemplo, como são aplicados os testes de significância dos coeficientes dos MLG, e quais são as estatísticas e os gráficos indicados para verificar a adequação do modelo. Ademais, nada foi encontrado na literatura sobre a identificação de modelos em experimentos fatoriais. Todos esses aspectos são detalhados nessa tese. Uma vez construído o modelo, é mostrado como usá-lo para obter as condições ótimas de operação dos processos e produtos. Além do cumprimento desse objetivo principal, a tese traz algumas contribuições adicionais; a saber: a) aponta limitações em todos quatro métodos da literatura que se propõem a escolher a transformação mais adequada para a resposta. Esses métodos não produziram resultados satisfatórios quando houve interações significativas entre os fatores; b) propõe a utilização de métodos de transformação da resposta como fonte de indicação da função de ligação a ser usada nos modelos lineares generalizados; e c) propõe a utilização da função de log-verossimilhança para uma escolha conjunta da distribuição de probabilidade e da função de ligação, nos modelos lineares generalizados.

\section{Palavras-chave}

Qualidade; Otimização de Processos; Modelos Lineares Generalizados. 


\begin{abstract}
Vieira, Antonio Fernando de Castro Análysis of Média e Dispersion in Unreplicated Factorial Experiments for the Optimization of Industrial Processes. Rio de Janeiro, 2004. 204 p. DSc Thesis - Department of Industrial Engineering, Pontifícia Universidade Católica do Rio de Janeiro.

This thesis puts together the statistical techniques indicated for modelling the mean and dispersion of quality characteristics of products and processes via unreplicated factorial experiments, resulting in the definition of an integrated and detailed script for the analysis. It was motivated by the fact that, although there are many publications about classic linear regression, generalized linear models (GLMs), response transformation and design of experiments, there is no one text which put together and describe in detail all the aspects of the modelling of the mean and the dispersion in factorial experiments. The few texts on the subject do not describe a number of important aspects in studies of this nature, e.g. how significance tests for the coefficients in GLMs should be applied and which are the statistics and plots indicated for checking model adequacy. In addition, nothing was found in the literature about model identification in factorial experiments. All these aspects are detailed in this thesis. Once the model is built, we show how to use it in order to obtain the optimal operating conditions for products and processes. Besides achieving this main objective, the thesis brings some additional contributions, namely: a) it points out limitations in all the four methods in the literature which have the purpose of selecting the most adequate transformation of the response; $b$ ) it proposes using response transformation methods as a source of indication of the link function to use in GLMs, and c) it proposes using the loglikelihood function for the joint choice of the probability distribution and of the link function in GLMs.
\end{abstract}

\title{
Keywords
}

Quality; Processes Optimization, Generalized Linear Models. 


\section{Sumário}

1 Introdução 15

2 Regressão Linear $\quad 26$

2.1 Estimação dos Parâmetros com Mínimos Quadrados 27

2.2 Testes de Hipótese em Regressão 30

2.3 Verificação da Adequação do Modelo 35

2.3.1 Análise dos Resíduos

2.3.1.1 Verificação da Suposição de Normalidade 35

2.3.1.2 Verificação da Suposição de Independência 41

2.3.1.3 Verificação da Suposição de Variância Constante 42

2.3.1.4 Verificação de Observações Atípicas 44

2.3.1.5 Verificação de Observações Influentes $\quad 47$

2.4 Otimização do Processo

2.4.1 Ponto Estacionário $\quad 51$

2.4.2 Ponto de Rendimento Máximo 53

2.5 Intervalos para a Média da Resposta e para a Previsão da Resposta 54

2.5 Mínimos Quadrados Ponderados 59

$\begin{array}{ll}2.6 \text { Forward Search } & 60\end{array}$

3 Modelos Lineares Generalizados 66

3.1 Distribuições de Probabilidade 66

3.2 Estrutura dos MLG 69

$\begin{array}{ll}3.3 \text { Estimação dos Parâmetros } & 71\end{array}$

3.3.1 Algoritmo para Estimar os Parâmetros 72

$\begin{array}{ll}\text { 3.3.1.1 Ciclo Interativo } & 75\end{array}$

3.4 Teste de Significância dos Coeficientes 76

3.4.1 Deviance

3.4.2 Estimação do Parâmetro de Dispersão

3.5 Adequação do Modelo

3.5.1 Resíduo Deviance 82 
3.5.3 Verificação da Função de Ligação 84

3.5.4 Verificação da Função de Variância 84

3.5.5 Distância de Cook

3.5.6 Forward Search 86

$\begin{array}{ll}3.6 \text { Quase-Verossimilhança } & 87\end{array}$

3.6.1 Estimação dos Coeficientes $\quad 89$

3.6.2 Estimação do Parâmetro de Dispersão 90

3.6.3 Significância dos Coeficientes $\quad 90$

$\begin{array}{ll}\text { 3.6.4 Adequação do Modelo } & 90\end{array}$

3.7 Quase-Verossimilhança Estendida 92

3.7.1 Estimação dos Coeficientes 93

3.7.2 Estimação do Parâmetro de Dispersão 94

3.7.3 Significância dos Coeficientes

3.7.4 Adequação do Modelo 94

3.8 Quase-Verossimilhança Restrita 94

4 Análise da Média $\quad 95$

4.1 Variáveis de Resposta 96

4.2 Estágio 1: Modelo Linear e Mínimos Quadrados (MQ) 99

4.3 Estágio 2: Transformação da Resposta 119

4.3.1 Definição da Transformação da Resposta 120

4.3.2 Estimação e Teste de Significância dos Coeficientes 129

$\begin{array}{ll}\text { 4.3.3 Testes de Adequação do Modelo } & 130\end{array}$

4.4 Estágio 3: Modelos Lineares Generalizados 147

4.4.1 Etapa 1- Distribuição de Probabilidade e Função de Ligação 150

$\begin{array}{ll}\text { 4.4.2 Etapa 2-Teste de Significância dos Coeficientes } & 157\end{array}$

$\begin{array}{ll}\text { 4.4.3 Etapa 3-Testes de Adequação do Modelo } & 158\end{array}$

5 Análise Conjunta da Média da Dispersão 170

5.1 A Relação do Modelo da Média com os Efeitos na Dispersão 171

5.2 Método Gráfico 172

$\begin{array}{ll}5.3 \text { Método de Box e Meyer } & 175\end{array}$ 
5.4 Métodos Iterativos - Modelagem Conjunta da Média e Variância

5.4.1 Método de Lee e Nelder

5.4.1.1 Modelo para a Média

181

5.4.1.2 Modelo para a Dispersão

183

5.4.1.3 Procedimento Iterativo

184

5.5 Análise da Média e da Dispersão e os Fatores de Ruído 190

$\begin{array}{ll}\text { 5.5.1 Fatores de Ruído } & 190\end{array}$

5.5.2 Fatores de Dispersão e Fatores de Ruído 194

5.5.3 Procedimento Geral - Fatores de Dispersão e Fatores de Ruído 195

5.6 Roteiro para Analisar a Média e a Variância 196

$\begin{array}{ll}\text { 5.6.1 Análise da Média } & 196\end{array}$

5.6.2 Análise Conjunta da Média e da Dispersão 197

6 Conclusões 198

$\begin{array}{ll}\text { Referências Bibliográficas } & 201\end{array}$ 


\section{Lista de figuras}

Figura 2.1 - Gráfico de Probabilidade Normal dos Resíduos 37

Figura 2.2 - Gráfico de Probabilidade Normal com Envelope 38

Figura 2.3 - Gráfico dos Resíduos Studentizados 41

Figura 2.4 - Gráfico Valor Absoluto dos Resíduos Versus Valor Ajustado 44

Figura 2.5 - Gráfico do Resíduo Outlier-t Versus Valor Ajustado 46

Figura 2.6 - Gráfico de Probabilidade Normal do Resíduo Outlier-t 47

Figura 2.7 - Gráficos de $y_{1}$ Versus $x_{1}$ e $y_{2}$ Versus $x_{2} \quad 48$

Figura 2.8 - Gráfico da Distância de Cook $\quad 50$

Figura 2.9 - Forward Search de $R^{2}$ e da Estimativa de $\sigma^{2} \quad 64$

Figura 2.10 - Forward Search dos Resíduos Padronizados 65

Figura 2.11 - FS das Estimativas dos Coeficientes e da Estatística de Teste $t \quad 65$

Figura 3.1 - Dependências Funcionais $\quad 74$

Figura 3.2 - Algoritmo MQPI 74

Figura 3.3 - Gráfico de Probabilidade Normal com Envelope 83

Figura 3.4 - Gráfico do Resíduo Studentizado Versus Valor Ajustado 84

Figura 3.5 - Valor Absoluto do Resíduo Studentizado Versus Valor Ajustado 85

Figura 3.6 - Gráfico da Distância de Cook 86

Figura 3.7 - Gráfico da $F S$ para a Deviance e $\hat{\phi} \quad 86$

Figura 3.8 - Gráfico da FS para o Resíduo Deviance 87

Figura 3.9 - Gráfico dos Resíduos Deviance Studentizados versus Valores

Ajustados (à esquerda) e Gráfico Valor Absoluto do Resíduo de Deviance

Studentizados Versus Valor Ajustado (à direita) 92

Figura 4.1 - Gráfico dos Efeitos - Modelo (M1) 102

Figura 4.2 - Gráfico dos Efeitos - Modelo (M2) 102

Figura 4.3 - Gráficos de Probabilidade Normal dos Resíduos. Modelos com o Fator C (esquerda) e sem o Fator C (direita) 104

Figura 4.4 - Gráfico dos Resíduos versus Valores Ajustados 104

$\begin{array}{ll}\text { Figura 4.5 - Distância de Cook } & 105\end{array}$

Figura 4.6 - Gráfico dos Resíduos Outlier-t 105 
Figura 4.7 - Gráfico Valor Absoluto do Resíduo Versus Valor Ajustado 106

Figura 4.8 - Gráfico dos Efeitos - Modelo Linear 107

Figura 4.9 - Gráfico de Probabilidade dos Resíduos 110

Figura 4.10 - Gráficos dos Resíduos versus Valor Ajustado 110

Figura 4.11 - Distância de Cook 111

Figura 4.12 - Forward Search de $\hat{\sigma}^{2}$ e de $R^{2} \quad 111$

Figura 4.13 - Forward Search dos Resíduos Padronizados 112

Figura 4.14 - Gráfico de Probabilidade Normal dos Efeitos 114

Figura 4.15 - Gráfico de Probabilidade Normal dos Resíduos com Envelope 115

Figura 4.16 - Gráfico dos Resíduos versus Valores Ajustados 115

Figura 4.17 - Gráfico Valor Absoluto do Resíduo versus Valor Ajustado 116

Figura 4.18 - Gráfico dos Resíduos Outlier-t 116

Figura 4.19 - Gráfico da Distância de Cook 117

Figura 4.20 - Dados do Exemplo 4.2: Forward Search de $\hat{\sigma}^{2}$ e de $R^{2} \quad 118$

Figura 4.21 - Forward Search dos Resíduos Padronizados 118

Figura 4.22 - Gráfico da Transformação de Box-Cox 122

Figura 4.23 - Gráfico Inverso para Valores de $\lambda \quad 124$

Figura 4.24 - Gráficos de Valor Ajustado versus Valor Ajustado 125

Figura 4.25 - Gráficos dos Valores Transformados $y(\lambda)$ versus $\hat{y}(1) \quad 126$

Figura 4.26 - Forward Search - Gráfico da Estatística de Teste 128

Figura 4.27 - Gráfico de Probabilidade Normal dos Efeitos 129

Figura 4.28 - Gráfico de Probabilidade Normal com Envelope 130

Figura 4.29 - Gráfico dos Resíduos Versus Valores Ajustados 131

Figura 4.30 - Gráfico dos Valores Absolutos dos Resíduos 131

Figura 4.31 - Gráfico dos Resíduos Outlier- $t \quad 132$

Figura 4.32 - Gráfico da Distância de Cook 132

Figura 4.33 - Dados do Exemplo 4.2: Forward Search de $\hat{\sigma}^{2}$ e de $R^{2} \quad 133$

Figura 4.34 - Forward Search dos Resíduos Padronizados 134

Figura 4.35 - Transformação de Box-Cox 136

Figura 4.36 - Transformação de Box-Cox - com as Interações Reais 136

Figura 4.37 - Gráficos Inversos para Valores de $\lambda \quad 137$

Figura 4.38 - Gráficos Inversos para $\lambda=1$ e $\lambda=-1 \quad 138$

Figura 4.39 - Gráficos de Valor Ajustado Versus Valor Ajustado 138 
Figura 4.40 - Gráficos dos Valores Transformados $y(j)$ Versus $\hat{y}(1) \quad 139$

Figura 4.41 - Forward Search da Estatística de Teste 140

Figura 4.42 - Forward Search da Estatística de Teste com Interações Reais 141

Figura 4.43 - Gráfico da Transformação de Box-Cox 143

Figura 4.43- Gráficos Inversos para valores de $\lambda \quad 144$

Figura 4.44 - Gráficos de Valor Ajustado Versus Valor Ajustado 145

Figura 4.45 - Gráficos dos Valores Transformados $Y(\lambda)$ Versus $\hat{Y}(1) \quad 145$

Figura 4.46 - Forwqrd Search da Estatística de Teste 146

Figura 4.47 - Gráficos de Probabilidade Normal dos Resíduos Deviance. À Esquerda: Distribuição Gama. À Direita: Distribuição Normal Inversa 158

Figura 4.48 - Gráfico dos Resíduos Deviance Versus Valores Ajustados. À Esquerda: Distribuição Gama. À Direita: Distribuição Normal Inversa

Figura 4.49 - Gráfico dos Valores Absolutos dos Resíduos Deviance Versus Valores Ajustados. À Esquerda: Distribuição Gama. À Direita: Distribuição Normal Inversa

Figura 4.50 - Gráficos com as Distâncias de Cook. À Esquerda: Distribuição Gama. À Direita: Distribuição Normal Inversa

Figura 4.51 - Gráficos de Probabilidade Normal dos Resíduos Deviance. À Esquerda: Sem Interação. À Direita: Com Interação

Figura 4.52 - Gráficos dos Resíduos Deviance Versus Valores Ajustados À Esquerda: Sem Interação. À Direita: Com Interação

Figura 4.53 - Gráfico do Valor Absoluto do Resíduo Deviance Versus Valores Ajustados. À Esquerda: Sem Interação. À Direita: Com Interação

Figura 4.54 - Gráficos dos Resíduos Deviance Versus Valores Ajustados: À Esquerda: Função de Ligação Logarítmica. À Direita: Função de Ligação Inverso

Figura 4.55 - Gráficos dos Resíduos Deviance Versus Valores Ajustados: À Esquerda: Distribuição Normal. À Direita: Distribuição Gama 168

Figura 4.56 - Gráfico dos Valores Absolutos do Resíduos Deviance Versus Valores Ajustados. À Esquerda: Distribuição Normal. À Direita: Distribuição Gama

Figura 5.1 - Gráfico dos Resíduos versus Fator C - Modelo (5.1)

Figura 5.2 - Gráfico dos Resíduos versus Fator C - Modelo (5.2) 
Figura 5.3 - Gráficos dos Resíduos Versus Fatores $F$ e $G$ - Modelo (5.2) 174

Figura 5.4 - Gráfico com os valores de $F_{j} \quad 177$

Figura 5.5 - Gráfico de Probabilidade Normal de $F_{j}$ - Modelo (5.1) 178

Figura 5.6 - Gráfico de Probabilidade Normal de $F_{j}$. Modelo (5.2) 178

Figura 5.7 - Gráfico dos Resíduos versus o Nível da Interação $A B \quad 179$

Figura 5.8 - Gráfico de POE versus $x_{2}$ e $x_{3} \quad 192$

Figura 5.9 - Gráfico de POE versus $x_{2}$ e $x_{3} \quad 193$

Figura 5.10 - Gráficos de POE e do Rendimento Versus Variáveis $x_{2}$ e $x_{3} \quad 193$ 


\section{Lista de tabelas}

Tabela 2.1 - Dados para o Modelo de Regressão Linear 28

Tabela 2.2 - Nível do Fator (Exemplo 2.1) 32

Tabela 2.3 - Dados Resultantes do Experimento (Exemplo 2.1) 33

Tabela 2.4 - ANOVA do Experimento (Exemplo 2.1) 33

Tabela 2.5 - Testes para os Coeficientes 34

Tabela 2.6 - Testes para os Coeficientes $\quad 34$

Tabela 2.7 - Testes para os Coeficientes 34

Tabela 2.8 - Resíduos Ordenados e Freqüência Cumulativa 36

Tabela 2.9 - Resíduo Ordenado e Quartil Normal 37

Tabela 2.10 - Dados “Ascomb” 47

$\begin{array}{ll}\text { Tabela 3.1 - Dados para o Modelo } & 69\end{array}$

Tabela 3.2 - Ligações Canônicas para os MLG 70

Tabela 3.3 - Dados do Experimento $\quad 80$

Tabela 3.4 - Estimativa dos Coeficientes e Erro Padrão 80

Tabela 3.5 - Análise de Deviance (ANODE) 81

Tabela 4.1 - Dados do Experimento de Moldagem 102

Tabela 4.2 - Estimativas dos coeficientes e Teste $t$ para o Modelo M2 103

Tabela 4.3 - Estimativas dos Coeficientes e Teste $t$ para o Modelo Linear 107

Tabela 4.4 - Estimativas das Respostas para o Modelo Linear e MLG-Gama 108

Tabela 4.5 - Dados do Experimento 109

Tabela 4.6 - Experimento da Perfuratriz 113

Tabela 4.7 - Teste $t$ para Experimento da Perfuratriz 114

Tabela 4.8 - Teste $t$ para Experimento da Perfuratriz 130

Tabela 4.9 - Dados do Experimento 135

Tabela 4.10 - Dados do Experimento 142

Tabela 4.11 - Função de Log-verossimilhança do Modelo Completo 156

Tabela 4.12 - Função de Log-verossimilhança dos Modelos Maior e Menor 156

Tabela 4.13 - Análise de Deviance (ANODE) 157

Tabela 4.14 - Valor da Função de Log-verossimilhança do Modelo Menor 164

Tabela 4.15 - Valor da Função de Log-verossimilhança 166

Tabela 4.16 - Valor da Função de Log-verossimilhança 167 
Tabela 5.1 - Cálculo dos Desvios-padrão dos Fatores $B$ e $C$

Tabela 5.2 - Estatística $F_{j}$ do Modelo (5.1) 177

Tabela 5.3 - Modelos e Componentes para a Média e a Dispersão 184

Tabela 5.4 - Experimento $2^{3}$ com Duas Replicações 185

Tabela 5.5 - Estimativa da Variância da Resposta 186

Tabela 5.6 - Coeficientes e Erro-Padrão do Modelo para a Dispersão 186

Tabela 5.7 - Coeficientes e Erro-Padrão do Modelo da Média 187

Tabela 5.8 - Coeficientes e Erro-Padrão do Modelo da Dispersão 187

Tabela 5.9 - Coeficientes e Erro-Padrão do Modelo da Média 188

Tabela 5.10 - Coeficientes e Erro-Padrão do Modelo da Dispersão 188

Tabela 5.11 - Experimento do Exemplo 5.1 191 